\section{Alas, porphyria}

\section{By Amy Donner, Senior Editor}

For over two decades, treatment of acute porphyrias has relied on hemin infusions that restore heme levels, but the therapy is slow to work, has side effects and is expensive. Now, a team led by Alnylam Pharmaceuticals Inc. has created an siRNA therapeutic that reduces disease symptoms in mice. ${ }^{1}$

However, to really improve patient care, the company will also need a strategy to raise awareness and combat misdiagnosis of these rare disorders.

Alnylam is completing IND-enabling toxicity studies. The company expects to submit an IND in 2014 and start clinical trials in 2015.

Acute hepatic porphyrias are orphan diseases caused by enzyme deficiencies that disrupt heme biosynthesis and ultimately result in misregulation of aminolevulinate synthase-1 (ALAS-1), the first enzyme in the heme biosynthetic pathway. These inherited disorders are characterized by life-threatening acute attacks that include severe abdominal pain, muscle weakness, seizures and paralysis.

According to Karl Anderson, the prevalence of acute porphyrias is unknown. "It is estimated at about 5 per 100,000. We usually see only those who are sick and not the many others who have not yet developed symptoms," he said.

Anderson is director of the Porphyria Laboratory \& Center and a professor in the Department of Preventative Medicine and Community Health at The University of Texas Medical Branch.

There are three known triggers of acute attacks: drugs that induce heme-dependent cytochrome P450 enzyme activity in the liver, hormonal changes, and starvation or extreme dieting.

Robert Desnick told SciBX that during an acute attack, patients often experience a prodromal period with brain fog and fatigue. Then, he said, "All hell breaks loose. They get excruciating abdominal pain that takes them to the emergency room."

However, the disease is often undiagnosed or misdiagnosed in the emergency room and mistaken for appendicitis because of the severe abdominal pain and a general lack of awareness about the condition.

Desnick is dean of genetic and genomic medicine and a professor and chair emeritus of genetics and genomic sciences at the Icahn School of Medicine at Mount Sinai.

Standard therapy for the attacks is i.v. infusion of hemin to restore heme levels. But patients often take a few days to respond, and because hemin cannot be stored in pharmacies, timely availability can be a problem.

In addition, transfusions are expensive, sometimes require an indwelling port and can damage veins because of the low solubility of hemin.

Recordati S.p.A. markets Normosang human hemin for treatment of hepatic porphyria. uniQure N.V. has the gene therapy AMT-021 in Phase I/II testing for porphyria. The therapy restores porphobilinogen deaminase, the enzyme most commonly disrupted by mutations that cause porphyria.

"Hemin has been a great drug, and we are grateful for it," said Desiree Lyon, executive director of the American Porphyria Foundation (APF). But, she noted, a new therapy would be welcome.

Hemin has been the mainstay of porphyria therapy because it provides exogenous heme that can restore feedback control over ALAS-1 expression. Overexpression of ALAS-1 during acute attacks causes accumulation of the metabolites 5-aminolevulinic acid (ALA) and porphobilinogen (PBG). Research over the last few years has shown ALA to be the primary mediator of the disease's neurotoxicity.

"Because all patients have a similar chemical presentation despite different genetic mutations, it was tough to figure out which metabolite was causing the trouble or whether heme deficiency itself was the culprit. We are more and more confident that the toxic molecule is ALA," said Montgomery Bissell, director of the Porphyria Center and a professor of gastroenterology at the University of California, San Francisco.

Thus, Desnick and his colleague Makiko Yasuda joined forces with Alnylam to test whether directly targeting the overexpression of ALAS-1 could prevent accumulation of ALA and PBG or restore normal metabolite levels and potentially provide a better therapy than hemin.

Yasuda is an assistant professor of genetics and genomic sciences at the Icahn School of Medicine.

\section{Running interference}

The team tested 45 siRNAs to find the most potent repressor of Alas- 1 mRNA expression in cultured mouse hepatocytes. The researchers then formulated the optimal siRNA, dubbed Alas1-siRNA1, in lipid nanoparticles to enable i.v. delivery to the liver.

In a mouse model of acute intermittent porphyria, prophylactic Alas 1-siRNA1 prevented upregulation of Alas-1 mRNA and accumulation of ALA and PBG in urine and plasma, whereas control siRNA did not. Protection against the acute attacks persisted for at least two weeks.

Next, the team compared how fast Alas1-siRNA1 and hemin produced their effects in a therapeutic setting. In the same mouse model, but after an attack was triggered, the siRNA reduced plasma and urine levels of ALA and PBG within 8 hours, whereas metabolite levels were stably elevated for at least 16 hours after hemin treatment.

Finally, the researchers looked at therapeutic and safety properties of Alas1-siRNA1. In the mice, the compound protected against neuromotor decline induced by repeated acute attacks and caused no change in liver alanine aminotransferase (Alt) and aspartate aminotransferase (Ast) levels, which are markers of liver function.

Furthermore, Alas1-siRNA1 did not lead to hepatic heme deficiency or affect the activity of the heme-dependent protein Cyp2e1 (cytochrome P450 family 2E1).

Bissell told SciBX that the demonstration that the siRNA does not deplete heme pools is particularly important. "You need to maintain heme production at a certain level to maintain function of heme proteins in the liver. Heme proteins turn over rapidly, and there is a lot of heme 


\section{ANALYSIS}

\section{COVER STORY}

cycling. You don't want to alter ALA production so much that you disrupt this."

The study was published in the Proceedings of the National Academy of Sciences.

"This paper provides preclinical proof of concept for our acute porphyria program," said Alnylam CEO John Maraganore. Alnylam is developing ALN-AS1, an N-acetylgalactosamine (GalNAc)-siRNA conjugate targeting ALAS-1 that can be delivered subcutaneously for the treatment of the acute hepatic porphyrias.

\section{Rapid response}

According to Bissell and the study's authors, one key benefit of the siRNA is its fast kinetics versus hemin.

Desnick and Yasuda said that although the siRNA reduced ALA and PBG levels faster than hemin, exactly why needs additional study. "Exogenous heme works primarily by decreasing ALAS-1 RNA production and by preventing the ALAS-1 protein from maturing. To the best of my knowledge, there are no studies characterizing how long each of these processes take to become fully functional and what the durability of each is in vivo," said Yasuda.

\section{"This paper provides preclinical proof of concept for our acute porphyria program."}

-John Maraganore, Alnylam Pharmaceuticals Inc.
For hemin, Bissell said, "it takes about three days before there is a clear biochemical response, and by day three or four, there is a rapid clinical response."

He added that there is often a lag in starting treatment because of delays in diagnosis.

"When we're talking about evaluating people in the emergency room, the key diagnostic test is urine PBG. But this is not available as an urgent test," said Bissell, and it can take up to a week to get the results. "People can sit for days without a diagnosis."

As with all orphan diseases, the low prevalence of porphyrias could present challenges in enlisting enough patients for clinical trials. In both the U.S. and Europe, networks of clinical centers focused on porphyria help recruit patients and manage trials for the disease. In the U.S., the Porphyrias Consortium is one of the NIH's Rare Disease Clinical Research Networks. The consortium includes five clinical centers in the U.S. and the APF. In Europe, the European Porphyria Network consists of 33 clinical centers in 21 countries and, like the APF, it aims to improve the diagnosis and treatment of porphyria.

Maraganore said that Alnylam plans to leverage the U.S. and European networks for its clinical trials of ALN-AS1.

Bissell also noted that Alnylam's subcutaneous formulation of the siRNA offers a significant treatment advantage over i.v. hemin as it would move treatment out of an infusion center and into the home, thus saving money and bypassing complications associated with the infusion itself. "This would be a major step forward," he said.

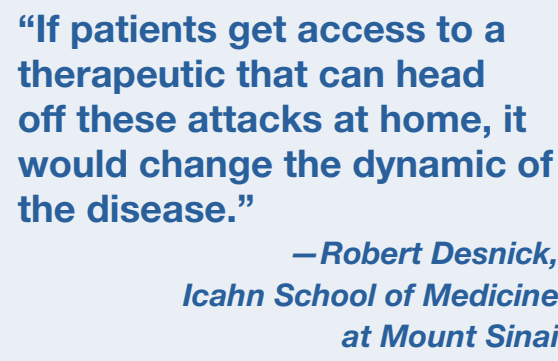

"If patients get access to a therapeutic that can head off these attacks at home, it would change the dynamic of the disease," said Desnick.

Alnylam and the Icahn School of Medicine have filed patents related to the findings. Alnylam has additional patents associated with its ALN-AS1 program. Method of use patents held by Mount Sinai are exclusively licensed to Alnylam.

Donner, A. SciBX 7(22); doi:10.1038/scibx.2014.631

Published online June 5, 2014

\section{REFERENCES}

1. Yasuda, M. et al. Proc. Natl. Acad. Sci. USA; published online May 12, 2014; doi:10.1073/pnas.1406228111

Contact: Robert J. Desnick, Icahn School of Medicine at Mount Sinai, New York, N.Y.

e-mail: robert.desnick@mssm.edu

Contact: Maria I. New, same affiliation as above e-mail: maria.new@mssm.edu2

COMPANIES AND INSTITUTIONS MENTIONED

Alnylam Pharmaceuticals Inc. (NASDAQ:ALNY), Cambridge, Mass.

American Porphyria Foundation, Houston, Texas Icahn School of Medicine at Mount Sinai, New York, N.Y. National Institutes of Health, Bethesda, Md.

Recordati S.p.A. (Milan:REC), Milan, Italy uniQure N.V. (NASDAQ:QURE) Amsterdam, the Netherlands University of California, San Francisco, Calif. The University of Texas Medical Branch, Galveston, Texas 